\title{
Frayling, Christopher: Mad, Bad and Dangerous? The Scientist and the Cinema
}

Reaktion Books, 2005. III, 239 S. Ill. £ 19.95. ISBN 1-86189-255-1.

Peter Lippuner

\section{Nur das Abnormale zählt}

Wissenschaftler werden selten normal dargestellt. Oft sind sie wie in vielen James-Bond-Filmen körperlich behindert: der Weltbeherrscher mit seiner eisernen Hand, sein kleinwüchsiger grausamer Assistent mit dem kindlichen Lachen. Dr Nos ${ }^{1}$ Handprothese findet ihre Entsprechung im Narbengesicht von Blofeld ${ }^{2}$ oder in den Metallzähnen von Beisser ${ }^{3}$. Wie Frayling zeigt, hat die Reduktion des Wissenschaftlers auf körperliche Behinderungen schon sehr viel früher angefangen. So ist in Siodmaks Donovan's Brain der Wissenschaftler nur noch auf sein Hirn reduziert, das in einem Glas in einer grauen Lösung schwimmt - und dennoch menschliche Gefühle zeigen kann. Noch weiter geht The Lady and the Monster: Das im Glas liegende Hirn will, von Rache getrieben, sich langsam die Kontrolle über einen Laborassistenten verschaffen. Und schliesslich The Man With Two Brains. Hier ist sogar eine Liebesgeschichte zwischen Annie, dem Gehirn, und dem Hirnchirurgen Dr. Hfuhruhurr möglich!

Äusserlich sehen sich die dargestellten Wissenschaftler verblüffend ähnlich, «The scientist as lab-coated, frizzy-haired, bespectacled sociopath» (Frayling 2005, S. 32). Aus unzähligen Filmen destilliert Frayling sechs Grundtypen des Kinowissenschaftlers heraus:

1 James Bond, Dr No, released 5th October 1962.

2 James Bond, You Only Live Twice, released 12th June 1967.

3 James Bond, Moonraker, released 26th June 1979.

Peter Lippuner, Geiselweidstrasse 6, CH-8400 Winterthur (Peter.lippuner@swissmedialink.ch). 
- alchemist (late 16th century), who seeks arcane forbidden knowledge, works in secret and alone or with a single assistant [...], is driven by a mania for power or gold and tends to be intellectually arrogant;

- absent-minded professor (mid-17th century), who is so obsessed with the single-minded pursuit of a tiny branch of knowledge [...] that he neglects his social or domestic responsibilities;

- inhuman rationalist (early 19th century), who has suppressed human affections and emotions in the cause of detached scientific enquiry [...] and who ignores wider moral dimensions of the results;

- heroic adventurer (late 19th century), who intrepidly goes where no man has gone before in the physical or intellectual world, who is not a complex character although he may well have personal eccentricities, and who classifies or lays claim to new territory;

- helpless scientist (mid-20th century), who is probably well-intentioned but whose discoveries behave in unpredicted ways or are hijacked by government/corporate interests;

- social idealist (mid-20th century, picking up on late H. G. Wells), whose idealism has less to do with hard research than with a social conscience or a search for wholeness, and whose maverick heroism emerges from non-compliance with government or industry. (S. 35f.)

Nach Frayling stützt sich die Alltagskultur auf eine überschaubare Galerie festverankerter Bilder, die seit einem halben Jahrtausend Literatur und später auch Filme mit den gleichen Stereotypien «beliefert». So findet der zerstreute Professor seine Entsprechung im «stupid virtuoso or amateur collector» (S. 36), der aus den Tagen der Royal Society stammt und heute noch in Comics weiterlebt. Der heroische Abenteurer hat seine Pendants in Jules Verne, den späteren Werken von H. G. Wells oder in den Challenger Stories von Conan Doyle und lebt heute ebenfalls noch in Comics oder in Filmen wie Starship Enterprise und Indiana Jones weiter. Am reinsten hat sich das Bild des Alchemisten gehalten: Isolation, Arroganz, überragende Hybris und unstillbarer Durst, mit allem zu experimentieren (von dem man als Wissenschaftler am klügsten die Finger lassen sollte), das zeichnet den alchemistischen Wissenschaftler aus. Und schliesslich kam im 2. Weltkrieg mit der Atombombe der neue Typus des hilflosen Wissenschaftlers auf, der später seine Fortsetzung im Zeitalter der Umweltverschmutzung und Genmanipulation fand.

Solche Typisierungen finden sich überall. Making Science our Own, eine detaillierte Untersuchung zur Darstellung von Wissenschaftlern in amerikanischen Populärmagazinen zwischen 1910 und 1950 teilt Wissenschaftler in der Literatur in vier Kategorien ein: Experte, Held, Magier und Schöpfer/ Zerstörer. Im Film kommt der Letztgenannte am häufigsten vor.

Unter mehr als tausend in Grossbritannien herausgegebenen Horrorfilmen zwischen 1931 und 1984 fand eine Studie folgende interessante Tatsachen:

- $31 \%$ der Bösewichte waren «mad scientists or their creations〉.

- Wissenschaftliche Forschungen waren der Grund für 39\% aller in den Horrorfilmen vorkommenden Drohungen. 
- Nur $11 \%$ der in den Horrorfilmen vorkommenden Wissenschaftler waren Helden (und das vor allem in Filmen vor 1960) (S. 41).

- In $74 \%$ der Horrorfilme war die Wissenschaft «the key organizing principle» (S. 41).

Von solchen Stereotypien geht eine Kraft mit klaren Auswirkungen aus. Als um den 11. September 2001 herum ein dreiminütiger Filmclip aus rund 110 amerikanischen Spielfilmen zusammengestellt wurde, um die Grösse und das Unvergleichliche der USA zu feiern, fand sich darin kein einziger «normaler» Wissenschaftler, abgesehen einmal von Charles Lindbergh's Spirit of St. Louis (1957) oder vom Weltraumfilm The Right Stuff (1983). Dafür kamen Wissenschaftler vor wie Dr Strangelove, The Nutty Professor (1996) oder Wissenschaftler aus angstschürenden Filmen wie The China Syndrome (1979) oder Erin Brockovich (2000). Die Haltung «Wir schaffen es> aus den 1930er Jahren und die Biographiefilme über amerikanische Wissenschaftlerinnen und Wissenschaftler aus den 1940ern schienen nicht mehr zum richtigen Stoff zu gehören.

Aus einer Auswahl von 16000 Spielfilmen zwischen 1910 und 1990 hatte Jacques Jouhaneau 520 Filme näher betrachtet. Er fand heraus, dass die Bösewichte in zeitlichen Wellen auftraten. So war die Blütezeit der Schurken unter den Wissenschaftlern in der Mitte der 1930er Jahre, dann erneut von 1940 bis 1945 und zuletzt in den späten 1970ern und frühen 1980ern. Davon waren $41 \%$ Mediziner, 35\% undefinierte Wissenschaftler («undifferentiated savants» [S. 42] wie Erfinder, Entdecker, Macher und Problemlöser). Mit $26 \%$ stellten Psychologen und Psychoanalytiker mehr als ein Viertel aller Übeltäter. Positiv besetzte Wissenschaftler, also Wohltäter an der Menschheit und anerkannte Forscher, kommen nur in 51 Filmen vor. Etwas weniger positive Kategorien, also die «sympathetic charlatans to sorcerer's apprentices across the board to assassins» (S. 43), kommen auf fast 200 aus einem Total von 520 Filmen.

Warum ist die Zahl an Bösewichtern unter den Wissenschaftlern eigentlich so hoch? Nach Fraylings Interpretation hat das Kino stets das Bild der gefährlichen Wissenschaft und menschenfeindlichen Technologie vermittelt. Dies mag Wissenschaftler zwar nicht gross stören, denn

\footnotetext{
Scientists [...] can state that the only meanings of science that matter to them are those that are firmly rooted in the interests and preoccupations of the scientific community to which they belong - that the only reading matter that counts is in lab reports or refereed scientific papers; [...]. (S. 44)
}

Damit stellt sich für Frayling zu Recht die Frage nach der Verantwortung des Wissenschaftlers für das veröffentlichte Bild. Kein Verständnis zeigt der Autor für Leute wie den Medizin-Nobelpreisträger Sir Peter Medawar, der 
sich verständnislos über die Ängste der Öffentlichkeit über Genmanipulation äusserte:

I find it difficult to excuse the lack of confidence which otherwise quite sensible people have in the scientific profession [...] for their fearfulness, laymen have only themselves to blame and their nightmares are a judgement on them for their deep-seated scientific illiteracy. (S. 44)

Wenn Frayling für einen intensiven und ehrlichen Dialog zwischen Wissenschaft und Öffentlichkeit plädiert, der auf die Ängste der Bevölkerung Rücksicht nimmt, dann versteht er darunter nicht einfach einen «rhetorical shooting-match» (S. 44). Der Physiker Brock Chisholm hat die Konsequenzen einer arroganten und herablassenden Haltung bereits in den späten 1950er Jahren in seinem Bulletin of the Atomic Scientists (vol. X, p. 355) vorausgesehen: «The human race may begin to fear its scientists to such an extent that it will take uncontrolled action towards them» (S. 45). Was für eine Aussage, gemacht mehr als ein Vierteljahrhundert, bevor Tierschutzaktivisten und Globalisierungsgegner zu mobilisieren begannen!

Gerade weil Stereotypien über «the mad scientist» nicht so schnell aus den Hirnen der Leute verschwinden, müsse der Wissenschaftler diesem Umstand in seinen Äusserungen und seinem Handeln kritisch Rechnung tragen, denn

[...] you almost don't notice he [the mad scientist] is there. All these debates about «creation science» versus «Darwin» are almost beside the point. The real creation myth of modern times is not Darwin, not Genesis; it is Frankenstein. (S. 47)

\section{Der «mad scientist> und die Politik}

Das Buch Mad, Bad and Dangerous? schlägt einen weiten Bogen von den ersten Filmstreifen des französischen Zauberers Georges Méliès über Fritz Langs Metropolis bis zu Eddie Murphy's Nutty Professor von 1996. Einer der ersten, die sich im Film mit wissenschaftlichen Themen befasst hatten, war der französische Zauberer und Theaterbesitzer Georges Méliès. Weil ihn optische Illusionen faszinierten, drehte er in Bahnhöfe einfahrende Züge, filmte Segelschiffe auf Flüssen und verfolgte Autorennen. Méliès wagte sich bald auch an die Umsetzung literarischer Stoffe und schuf nach dem Vorbild von Jules Verne und H. G. Wells 1902 den Film Le Voyage dans la Lune und drei Jahre später Le Voyage à travers l'Impossible. Im ersten Film liessen sich Astronauten von einer Kanone auf den Mond schiessen, im zweiten reisten wagemutige Abenteurer in einem fliegenden Zug zur Sonne und tauchten dort in den glühenden Ball ein. Fast wären sie in der feurigen Glut umgekommen, wenn nicht der Filmheld die Reisenden wieder heil auf die Erde zurückgebracht hätte, wo sie im kühlen Ozean ein langersehntes Bad fanden. 
Méliès begeisterte sich für die technologische Entwicklung. Er drehte Ballonaufstiege, machte Filme über Elektrizität, drehte Autorennen und ganz besonders interessierte ihn die Röntgenstrahlung. Seine Wissenschaftler kamen meist als hyperaktive, mehr oder weniger exzentrische Gelehrte daher. Méliès' Interesse für die Wissenschaft war so gross, dass er sofort auf Neuerungen reagierte. Einsteins erstmalige Spekulation über die Krümmung von Raum und Zeit veranlasste ihn, allein in einem Jahr 22 Filme zu diesem Thema zu produzieren. Gleichzeitig schufen die Thomas-A.-Edison-Studios in den USA erstmals auch wissenschaftliche Trickfilme wie $A$ Trip to Mars und Edison's Conquest of Mars. Auch hier dasselbe Bild des Wissenschaftlers: «a wizard of science who can't help inventing things» (S. 56).

Die 1920er Jahre veränderten das Bild des Wissenschaftlers im Film erneut. Im Film Metropolis stellte Fritz Lang seinen Wissenschaftler als eine Art Magier dar. Dr. Rotwang, der Filmheld, sollte aus einer Frau einen Roboter machen. In vielem unterschied sich der Film von den damals üblichen Produktionen, vor allem glänzte er durch eine brillante Lichtführung. In Metropolis war ein Produkt über den Kampf moderner Wissenschaft gegen Okkultismus entstanden, ein Kampf, in dem der Magier Dr. Rotwang zum Bösen schlechthin wurde. Äusserlich blieb Rotwang zwar ein moderner Wissenschaftler mit einem Labor in Metropolis, einer modernen von hohen Wolkenkratzern und weiten Strassenschluchten dominierten Stadt. Im Laborinneren dagegen widerspiegelte der Film die jahrhundertealte alchemistische Tradition des Mittelalters überdeutlich:

[...] his laboratory-workshop is filled with retorts on stands, neon tubes, curved vessels, diagonal electrical cables across the ceiling, arrows and chevrons and much bubbling glassware - but in the fireplace there are some bellows, like at an alchemist's and a stuffed crocodile would not be out of place. Rotwang himself has unruly white hair, and a black metal right hand - the result $[\ldots]$ of some unnamed laboratory accident. (S. 61)

Doch die Reaktion auf Metropolis war zwiespältig. In England stiess der Film auf völlige Ablehnung. Er sei eine Anhäufung von Platitüden und Klischees, so die Kritik. Vor allem H. G. Wells fand den Film besonders schlecht und hielt ihn für

a muddlement about mechanical progress and progress in general served up with a sauce of sentimentality that is all its own [...]. (S. 76)

Sieben Jahre später produzierte er dann seinen eigenen Film Things to Come. Dabei gelobte er, alles besser zu machen. Seine Anweisungen an das Design Team enthielten unter anderem Folgendes:

All the balderdash one finds in such a film as Fritz Lange's [sic] Metropolis about robot workers and ultra skyscrapers etc should be cleared out of your minds before you work on this film. As a general rule you may take it that whatever Lange did in Metropolis is in the exact of what we want done here [...]. (S. 77) 
Doch Wollen und Können sind bekanntlich zweierlei. Der Film entsprach keineswegs Wells' Erwartungen und Wünschen. Er erkannte, dass Literatur und Drehbücherschreiben zwei verschiedene Dinge waren. Was ihm leicht aus der Feder floss, war oft schwerfällig in der Filmumsetzung.

So wurden Film und Wissenschaft immer mehr zu zwei eng miteinander verknüpften Bereichen. Dies zeigte sich besonders nach dem Zweiten Weltkrieg in den USA. Dort suchte der deutsche Raketenspezialist Wernher von Braun finanzielle Mittel für sein eigenes Weltraumprogramm. Er wandte sich dabei an den Filmemacher Walt Disney. Disney hatte von Brauns Beschreibung einer Weltraumstation gelesen und war sofort begeistert. Er wollte den Weltraum zu einem zentralen Thema in einem seiner Erlebnisparks machen. Vor allem aber suchte er Material für seine wöchentlichen Fernsehprogramme: Der Weltraum und von Braun kamen ihm dabei sehr gelegen.

He was a wonderful character to work with - great sense of humour. He fitted right into the Disney setup. He seemed to have all the facts and figures [...]. (S. 91)

Time Magazine nannte ihn gar «the major prophet and hero (or wild propagandist, some scientists suspect) of space travel» (S. 91). So entstanden Filme wie Man in Space und Man in the Moon. Nebenbei plante von Braun eine $25 \mathrm{~m}$ grosse Rakete für Disneys Erlebnispark in Anaheim. Diese wurde zur grossen Attraktion und zum weithin sichtbaren Anziehungspunkt des Parks.

Von Braun wollte aber mehr, viel mehr. Ihm war klar - nur über den Film konnte er die amerikanische Bevölkerung für sein eigenes Weltraumprogramm interessieren. «I have the chance to talk to the great American public through television and film» (S. 98), sagte er einmal und fügte an, dass Volk und Regierung auf diesem Wege «more rocket conscious» (S. 98) gemacht werden könnten. Von Braun wollte den Sowjets etwas Ebenbürtiges entgegenhalten. Mit I Aim at the Stars gelang ihm ein erster, zwar unkritischer und die Weltraumfahrt verherrlichender Film, ein Film, der gerade in Grossbritannien schlecht ankam. Dort hielt man ihm vor, er hätte sich um die militärische Seite der Raketen foutiert und die Verwüstungen nicht erwähnt, die die V2 (also von Brauns Raketen) gerade in London im Krieg verursacht hätten.

\section{Ist Frankenstein überall?}

Die Zeit für eine solche Figur war Anfang der 1930er Jahre reif geworden, denn in all den Krisenjahren hatten sich Wissenschaftler immer wieder in 
ihren Voraussagen über die Entwicklung der Wirtschaft in den USA geirrt. So war es denn kein Wunder, dass die Öffentlichkeit den Äusserungen der Wissenschaft gegenüber nur wenig Glauben schenkte. Die Darstellung des Wissenschaftlers Henry Frankenstein im Film von James Whale als «mad scientist , als verrückter Wissenschaftler, war deshalb nur folgerichtig. Immer mehr <mad scientists t tauchten in grosser Zahl und in vielen Varianten auf. Entgegen dem literarischen Original ist Henry Frankenstein von der übrigen Wissenschaftlergemeinde jedoch völlig isoliert, seine Tätigkeit ist sinnlos, ja sie trägt nichts zur Verbesserung der Gesellschaft bei. Das ohnehin stark vorhandene Misstrauen gegenüber Intellektuellen tat das Seine dazu. Wissenschaft «is expected to be not only comprehensible, but practical as well» (S. 117). Diesen Grundsatz sahen viele Leute verletzt. Wer die von Gott und der Natur gesetzten Grenzen überschreitet, ist eben «evil». Und genau das hatte Henry Frankenstein mit seinem Monster getan.

Der Film mit Boris Karloff als Monster war ein Kassenerfolg. Gleich mehrere Filme ähnlichen Inhalts folgten rasch: $D r$ X, Mystery of the Wax Museum, Murders in the Rue Morgue, Son of Frankenstein, Dr Jekyll and Mr Hyde oder The Mask of Fu Manchu. Sogar Walt Disney sprang mit seinem Trickfilm The Mad Doctor auf den Zug auf. Wie sich der Geschmack im Verlaufe der Zeiten aber ändern kann, zeigt die Tatsache, dass in den 1960er und 1970er Jahren diese Thematik immer wieder auch in Komödien und Parodien vorkommt. James Whales Frankenstein wird zu Munster, Go Home! (1966), Dr Jekyll zum Nutty Professor (1996), Dr X und Rue Morgue finden sich wieder in The Man With Two Brains (1983). Frankenstein war überall. Doch die Gegenreaktion blieb nicht aus. Hollywood begann in den frühen 1930er Jahren, nicht nur verrückte Wissenschaftler darzustellen, sondern hielt auch bewusst Ausschau nach wissenschaftlichen Grössen, die etwas Positives und für die Gesellschaft Wertvolles unternahmen. Man ist geneigt zu sagen, dass die «evil scientists» nach «saintly scientists» (S. 166) riefen, zum Beispiel nach The Story of Louis Pasteur (1936), Dr Ehrlich's Magic Bullet (1940), The Story of Alexander Graham Bell (1940) oder nach Madame Curie (1943). Diese Filme schufen gleichsam einen neuen Typ Filmwissenschaftler, sozial engagiert und bereit, seine Arbeiten in den Dienst für die Öffentlichkeit zu stellen. Im Gegensatz zum «mad scientist〉 soll dieser Wissenschaftler ehrenvolle Motive haben, er riskiert für die Forschung sein eigenes Leben, ist unbürokratisch und interessiert sich weder für Geld, Macht noch Prestige. Ehrlich sagt in seinem Film Dr Ehrlich's Magic Bullet die berühmten Worte:

There can be no final victory over the diseases of the body, unless the diseases of the soul are also overcome. They feed upon each other [...]. In days to come, there will be epidemics of 
greed, hate, ignorance. We must fight them in life as we fought syphilis in the laboratory. Fight, fight, fight. We must never stop fighting. (S. 142)

Dass solche Hollywoodglorifizierungen oft nicht der Realität entsprachen, lässt sich leicht am Film Madame Curie überprüfen. Aus dem zu einer veritablen Liebesgeschichte gewordenen Film erfährt man beispielsweise nicht, dass Marie Curie auch Beraterin der Radium-Bergbauindustrie war und ihre Reisen nach Amerika hauptsächlich dazu nutzte, auf Radiumbasis hergestellte Gegenstände des Alltags zu propagieren (z.B. Radium-Cocktails, Radium-Lippenstifte, Radium-Kuren etc.). Dennoch zeigte der Film vielleicht zum ersten Mal in der Geschichte Aspekte, die dem wirklichen Wissenschaftlerleben viel näherstanden. Dazu ein Kritiker der Times:

What makes Madame Curie a film of rare distinction is not that the lady discovered radium: it is the revelation of a modern saint. No monk in hair shirt ever mortified himself more painfully [...]. We have had scientific films that were livelier and more superficially exciting, but none that brought out so successfully the grinding fatigue of research work and the essential spirituality of those who devote their lives to explaining the material. (S. 162)

Madame Curie war auch in anderer Hinsicht ungewöhnlich. Der Film war einer der ganz wenigen, der einer Wissenschaftlerin den ihr gebührenden Platz zukommen liess. Normalerweise hatten Frauen in Filmen über Wissenschaft in dieser Zeit ohnehin nichts verloren. Sie waren entweder weissgekleidete, brillentragende Laborantinnen oder im schlimmsten Falle bloss Anhängsel des Wissenschaftlers, wie zum Beispiel in The Bride of Frankenstein. Ein eigenes Urteil hatten sie sich schon gar nicht zu erlauben. Dazu Telly Savalas im Film The Interns, wo er als Dr Riccio zur Chirurgin Dr Mado Bruckner (Haya Harareet) sagt:

It will be a long time before you are entitled to so weighty a thing as a judgement. Your errors are due to intuition: excusable in a woman but not in a doctor. (S. 164)

So einfach scheint das zu sein! In den 1950er Jahren machte sich ein weiteres Thema in den Horrorfilmen breit: die unerwarteten Folgen der Atomenergieforschung wie in The Invisible Ray (1936), Dr Cyclops (1940), Tarantula (1955), The Creature with the Atomic Brain (1955), Gamma People (1956), The Fly (1958) oder The Alligator People (1959). Mutierte Tiere, verkrüppelte Monster und radioaktive Zombies dominierten die Streifen. Von nun an wimmelte es in billigen Hollywoodfilmen der 1950er Jahre nur so von

giant ants, or grasshoppers or spiders or flies or scorpions or daddy-long-legs or mantises or reptiles or moles or cats or crabs or tree stumps, which threatened Eisenhower's America and which required the military (a new element in such movies) to destroy them. (S. 198)

In den 1970er Jahren kamen dann noch Umweltschäden und Seuchen dazu, die sich als Konsequenz solcher Forschung plötzlich wild ausbreiteten. Natür- 
lich blieb dies nicht ohne Folgen für das Image der Wissenschaftler und ihrer Organisationen: Erneut repräsentierte der Wissenschaftler das Böse. Unbewusst wurde das Bild in der Öffentlichkeit dadurch noch verstärkt, dass Wissenschaftler sich im wirklichen Leben immer wieder begeistert, um nicht zu sagen, unkritisch zu den Folgen ihrer Entdeckungen äusserten. So wurde die Radioaktivität beispielsweise zum

philosopher's stone, capable of creating unimagined wealth and of acting as the inexhaustible elixir of life [...] [and it] could make the whole world one smiling Garden of Eden. (S. 171)

Das Vokabular in solchen Filmen fand auch Eingang in die Politik. Als Amerika einen Verteidigungsschild gegen feindliche Raketen im Weltall aufbauen wollte, bediente sich Präsident Ronald Reagan hemmungslos der Sprache früherer Horror- oder Spionagefilme wie Murder in the Air (1940) oder Torn Curtain (Hitchcock, 1966). So würde der «inertia projector» (S. 172) die Vereinigten Staaten von Amerika unbesiegbar und das Weltraumsystem Gamma 5 «nuclear defense obsolete» (S. 173) machen. Sogar Präsident Reagans Berater staunten nicht schlecht über die Ähnlichkeit der Wortwahl zwischen solchen Filmen und der berühmten Rede ihres Präsidenten im März 1983, als er die Wissenschaftler des Landes aufrief:

I call upon the scientific community in our country, those who gave us nuclear weapons, to turn their great talents now to the cause of mankind and [...] peace, to give us the means of rendering nuclear weapons impotent and obsolete. (S. 172)

Die Rede wirkte. Der Kongress bewilligte eine erste Tranche von 26 Milliarden und erhöhte sie später auf 40 , «even though the programme had much more to do with science fiction than science fact» (S. 174).

\section{Abkehr vom <mad scientist〉?}

Freyling ist nicht der erste, der Wissenschaftlern, Alchemisten und anderen Zauberern nachsteigt. Margaret Mead hat schon 1957 Kinder Wissenschaftler zeichnen lassen, und Peter Weingart hat z.B. 2000-2002 im Rahmen des Bielefelder Forschungsseminars «The Perception and Representation of Science by Hollywood» über 220 Filme analysiert. Fraylings Übersicht über die Filmgeschichte fand trotzdem weite Beachtung. Vielleicht liegt es am Schreibstil des Londoner Sergio-Leone-Biographen und Italo-Western-Kenner, dass sein Buch eine wahre Fundgrube für den Cineasten ist. In seiner Untersuchung hat er die Information mehrerer Tausend Filme untersucht, und dabei überschwemmt er den Leser förmlich mit Fakten, von denen man wünschte, dass sie manchmal etwas besser eingeordnet worden wären. So 
fällt es immer wieder schwer, die grossen Linien der Filmgeschichte zu sehen. Allzuoft verliert er sich in - zwar spannenden - Details. Eine prägnantere Herausarbeitung, wie sich das Bild des Filmwissenschaftlers über die Jahre hinweg entwickelt hat, wäre leicht auf Kosten etwas weniger Information machbar gewesen. Doch Fraylings Verdienst tut dies keinen Abbruch. Vielmehr zeigt er, dass die alten Stereotypien immer wieder von neuem aktiv sind.

In einer Untersuchung an Schulen in Südwestengland liess Frayling Primarschüler spontan Wissenschaftler zeichnen - mit erstaunlichen Resultaten. Keiner der Knaben hatte eine Wissenschaftlerin gezeichnet, im Gegensatz zu den Mädchen, von denen 50\% genau dies getan hatten. Auf den Zeichnungen der Mädchen lächelten Wissenschaftlerinnen und Wissenschaftler viel mehr. Dafür kreierten die Knaben mehr verkrüppelte Wissenschaftler (Prothesen, Rollstuhl, grosse Ohren, ein Auge grösser als das andere). Beide Geschlechter zeichneten jedoch ihre Wissenschaftler mit Bleistiften und Reagenzröhrchen in den Taschen des Labormantels. Oft waren sie unrasiert und trugen ihr Haar wild auf alle Seiten gerichtet. Bunsenbrenner, Pipetten, Retorten und Test-Röhrchen gehörten zum Laborinventar - und nur auf einer Zeichnung fand sich auch ein Computer! Die alten Klischees scheinen nach wie vor lebendig zu sein, obwohl in den befragten Schulen weder das Lehrpersonal Labormäntel trägt, noch Physikoder Chemielabors vorhanden sind. Offensichtlich haben die Primarschüler eine genaue Vorstellung, wie ein Wissenschaftler auszusehen hat. Woher stammen diese Bildvorstellungen? Die Antwort liegt auf der Hand:Cartoons, Computerspiele und Komödien nehmen die alten Klischees nur allzugerne immer wieder auf.

Ist damit das Wissenschaftlerbild auf alle Ewigkeit fest zementiert? Eine leise Hoffnung, dass es auch anders werden könnte, sieht Frayling in den auf allen Sendern laufenden wohlbekannten Ärzteserien. Diese erwecken in ihm Hoffnung. Gleichsam als Beleg zitiert er den amerikanischen Physiker Spencer Weart mit folgenden Worten:

\footnotetext{
It would be nice if scientists were shown as people with outside interests, people who are not repressed, not odd. And this is being done in some movies. When you talk about ordinary people with ordinary emotional problems but who approach matters scientifically, who experiment, who solve problems - television doctors (in dramas) are exactly that. They are approaching problems scientifically - they are real scientists and yet they are also ordinary people. [...] that's the way to a certain extent they are portrayed in these hospital shows: "what's this person's problem, how do we find out, run lab tests, well it's not this let's try that"; that is what scientists do. (S. 225)
}

In der Tat, es wäre schön, wenn diese Hoffnung eines Tages wahr würde. 


\section{Unsichtbares sichtbar machen. Ungewöhnliche Einblicke in die Natur}

Allen drei ist eines gemeinsam, sie wollen von blossem Auge unsichtbare Phänomene in der Natur auf ihre spezielle Weise dem Betrachter visuell erschliessen: der Basler Martin Oeggerli mit dem Rasterelektronenmikroskop, der Münchner Astrophysiker Harald Lesch mit seiner kraftvollen Sprache und der Winterthurer Biologe Jakob Forster mit Fotoapparat und Filmkamera.

\section{Ein Micronaut auf Reisen - oder Kunst und Wissenschaft Dr. Martin Oeggerli, www.micronaut.ch}

Tulpenpollen, Spinnenhaare, Spiralmooskapseln, Fruchtfliegenaugen oder Darmbakterien - das sind nur ein paar der Lieblingsobjekte des Basler Biologen Martin Oeggerli. Bereits haben es einige seiner Bilder auf die Titelseiten der englischen Wissenschaftszeitung $E M B O$ gebracht, letztes Jahr wurde sein Bild über Rostpilzsporen gar zum besten «Scientific Image 2008» erkoren.

Abb.1. Spiralmooskapsel. REMAufnahme von Martin Oeggerli. (C) Martin Oeggerli 2006/Www. Micronaut.ch, powered by Prüftechnik Uri GmhH.

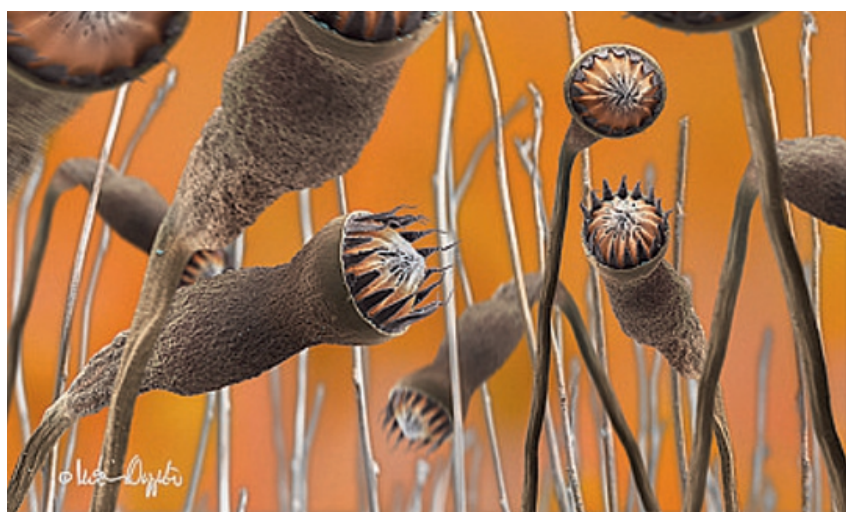

Martin Oeggerli ist fasziniert von den gestalterischen Möglichkeiten des Rasterelektronenmikroskops (REM). Mit dem Mikroskop taucht er in Bereiche ein, die dem menschlichen Auge völlig verborgen sind. «Im winzig Kleinen warten tausend Dinge darauf, entdeckt zu werden», sagt der junge Mann mit leuchtenden Augen. «Mit einem solchen Hightech-Gerät lassen sich Strukturen 10- bis 500000 -fach vergrössern und verblüffend plastisch abbilden $»^{4}$. Und in der Tat, seine Bilder gleichen Bergen und Tälern, fein-

4 Aus Schweizer Familie, «Mikro-Faszination» von Andrea Strässle. Aus dem Internet: www. schweizerfamilie.ch/wissen/natur/article/24167.html. 
ziselierten Lanzen und mikroskopisch kleinen Wäldern, jedes Bild ist ein Kunstwerk für sich.

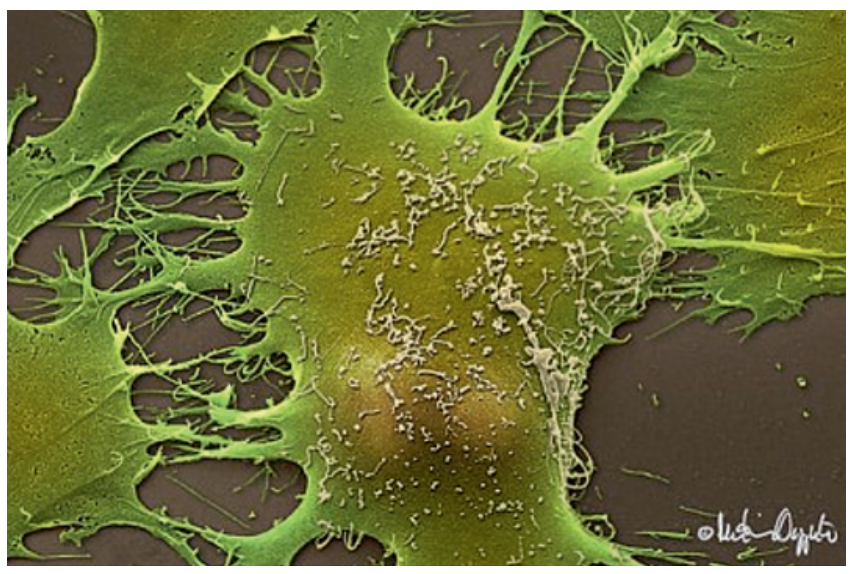

Abb. 2. Rattenzelle. REM-Aufnahme von Martin Oeggerli. (C) Martin Oeggerli 2006/www. Micronaut.ch, powered by Prüftechnik Uri GmhH.

Martin Oeggerli hat in Basel Biologie studiert. Heute arbeitet er zu 50\% in der Krebsforschung am Universitätsspital Basel. Nebenbei ist er aber ein begeisterter Fotograf. Da ein REM Hunderttausende von Franken kostet, Oeggerli sich ein eigenes also nicht leisten konnte, machte er aus seiner Not eine Tugend und suchte einen Partner. Diesen fand er im Forschungslabor der Prüftechnik Uri GmbH (kurz PTU), die einen wissenschaftlichen Leiter suchte. Oeggerli half der PTU mit seiner Forschungserfahrung, dafür durfte er im Gegenzug das Mikroskop für seine eigenen Aufnahmen brauchen. Organische Stoffe lassen sich nicht ohne weiteres im REM fotografieren. Zuerst muss der Untersuchungsprobe das Wasser entzogen und sie chemisch fixiert werden. Proben, die unter dem Mikroskop im Vakuum untersucht werden, müssen nämlich völlig trocken sein. Dann bedampft Oeggerli diese mit einer dünnen Gold- oder anderen Edelmetallschicht. Jetzt ist die Probe für die Untersuchung bereit. Ein Elektronenstrahl tastet die Gegenstände ab. Aus den reflektierten Elektronen entsteht somit ein Bild von grösster Genauigkeit. Vorläufig ist dieses aber noch schwarzweiss. Mit dem Computer färbt Oeggerli seine Schwarzweiss-Vorlagen anschliessend in stundenlanger Kleinarbeit naturgetreu ein. Ausnahmsweise können dabei bis zu 100 Stunden verstreichen. So sagt Oeggerli über ein Bild von Spiralmooskapseln:

In reality the moss forest was brown, but the orange background and tiny blue spores are my own design [...]. I tried to design the picture such that if you look at it, you think you are in a moss forest. ${ }^{5}$

Für Oeggerli birgt jedes Foto ein neues Rätsel. Auf den Aufnahmen findet er Details, die selbst den Naturwissenschaftler ins Staunen versetzen. Warum

5 «A journey into the world of the invisible», Swissinfo, 28.5.2008: http://www.swissinfo.ch/eng/ search/Result.html?siteSect $=882 \&$ ty $=$ st\&sid $=9144463$. 
ist der Schmetterlingsflügel fast ziegelförmig angelegt; was haben die Härchen zwischen den Einzelaugen der Fruchtfliege für eine Funktion; wie ist das Wasserfarnblatt aufgebaut, damit es nicht absinkt? Oeggerlis Bilder sind Zeugnisse der grossen Vielfalt der Natur und eignen sich dank hoher ästhetischer Wirkung hervorragend für eine Ausstellung oder als grossformatige Illustrationen. «Meine Bilder sollen mikroskopisch kleine Sachverhalte aus dem Bereich Medizin und Forschung ästhetisch sowie wissenschaftlich korrekt darstellen und dabei den Betrachter in unbekannte Welten entführen» ${ }^{6}$. Das gelingt Martin Oeggerli, dem künstlerischen Wissenschaftler, in der Tat mit jedem einzelnen seiner Werke.

\section{Alpha Centauri - Science Hit am TV. Wissenschaftssendung auf dem Kanal des Bayrischen Rundfunks BR: jeweils Mittwoch 22.45, im Internet abrufbar unter: http://www.br-online.de/br-alpha/alpha-centauri/alpha- centauri-videothek-2007-ID120843829435.xml}

Die Wissenschaftssendung «Alpha Centauri» des Bayrischen Rundfunks gehört vermutlich zu den optisch ärmsten in der weiten Fernsehlandschaft Europas! Vier Holzschulbänke, eine schwarze Wandtafel, im Hintergrund auf dem Blue Screen ein Sternsujet und im Verlaufe der 15-minütigen Sendung als einziges, zusätzliches optisches Element eine Fotografie, die das zentrale Thema beschreibt-fertig. Doch nein - ein wichtiges Element kommt in der Person von Professor Harald Lesch noch dazu, dem theoretischen Astrophysiker an der Ludwig-Maximilians-Universität in München, Philosophen und gleichzeitig Fernsehmoderator. Wenn Lesch jeweils zu später Stunde am Bildschirm auftritt, dann beginnt eine TV-Präsentation vom Feinsten. Ein kurzer, sprachlich explosiver Einstieg - und Lesch legt dar, worüber er in der nächsten Viertelstunde sprechen wird. Dann, nach einem kleinen Gang zur Wandtafel, schreibt er, ständig weiterreferierend, die der heutigen Sendung zugrundeliegende Frage an die Tafel. Und schon ist die einzige Aktion, die die Sendung kennt, auch schon vorbei - nach nur 50 Sekunden. Lehrer Lesch wird zum vertrauten Freund, der jetzt aber erst recht losfährt und Vollgas gibt. Das Zuhören wird zur wahren Freude - seiner Sprache und seiner Gestik wegen. Kein Wunder also, dass sich der Zuschauer nach wenigen Minuten völlig in Leschs Bann gezogen fühlt.

Das Rezept der Sendung ist denkbar einfach. Man nehme einen sprachgewandten Astrophysiker und lasse ihn über Astronomie reden. Sie haben

6 Aus Schweizer Familie, «Mikro-Faszination» von Andrea Strässle. Aus dem Internet: www. schweizerfamilie.ch/wissen/natur/article/24167.html. 
richtig gelesen - reden. Und das im Zeitalter des modernen Fernsehens. Wie kam es zu dieser Situation? Lesch in einem Interview:

Als wir in Nürnberg die erste Sendung gemacht haben, kam irgendwann von der Regie der Ruf: «So geht das aber nicht!» - Was war passiert? Nun, die Kameraleute, die eigentlich hinter ihren Geräten stehen sollten, standen plötzlich neben ihrem Equipment und hörten mir mit offenen Mündern zu. Die waren von dem Thema Schwarze Löcher und der Art und Weise, wie es vermittelt wurde, so fasziniert, dass sie die Kameraführung für einen Moment glatt vergassen. Da haben wir gemerkt, dass dieses Konzept auch bei dem Zuschauer ankommen muss. ${ }^{7}$

Und so redet er daher über schwarze Löcher, Higgs-Teilchen, Barionen, Big Crunch und was vor dem Big Bang alles war, redet, als ob es auf dieser Welt nichts Einfacheres gäbe. Immer sind es simple Fragestellungen mit ernstem wissenschaftlichem Hintergrund. Der Herr Professor geht sie dabei ohne eine einzige Grafik, ohne Simulationen und Tricks an. Sein Hilfsmittel ist einzig die Rhetorik, seine oft kurzen, bald wieder länger werdenden Sätze, das stets variierende Sprechtempo, die Stimme, manchmal lauter, um Sekunden später plötzlich wieder zusammenzubrechen. Das ist Lesch, ein Wissenschaftler voll Witz und Humor, der mit geheimnisvollem Augengezwinker dem Zuschauer oft fast flüsternd die neusten Fakten aus der Astronomie vermittelt. Kein Stottern, kein Ächzen - obwohl es so ist, als würde Lesch seine Sendungen aus dem Stand heraus direkt vor dem Zuschauer entwickeln. Denkpausen gehören da dazu, genauso wie seine wilde, aber stets Sinn machende Gestik. Seine Sprache ist diejenige des Menschen auf der Strasse, das Denken assoziativ und voller Gedankenblitze. Dabei weicht er nie von der grossen Linie ab. Instinktiv merkt Lesch, wenn er nicht verstanden werden könnte. Er bricht den Satz ab, setzt neu an und redet sich elegant aus jeder kommunikatorisch gefährlichen Situation heraus. Und ganz besonders wichtig für den Betrachter:Nie erhält man den Eindruck, Lesch hätte auf der Suche nach Verständlichkeit unhaltbare, die Fakten verfälschende Kompromisse geschlossen. Er drückt sich deshalb auch nie vor schwierigen Themen, die obigen Fragestellungen sind ein beredter Beweis dafür.

Lesch ist ein begeisterter und begeisternder Moderator; wenn seine Augen funkeln, sitzt man als Zuschauer vor dem Fernsehschirm, staunt und vergisst, den Mund zu schliessen. Ist so was wirklich möglich? Wissenschaftsvermittlung ohne Fachjargon und gelehrtes «Es tut mir leid, wenn du das nicht weisst»-Wissen, bei dem jeder für klug gehalten wird und jeder, der will, das Präsentierte auch verstehen kann? Ja, das ist möglich mit Lesch, einem Wissenschaftler, dem man glaubt, was er sagt, und der kompetent ist. Bekanntlich liegt Kompetenz ja nicht im Gebrauch möglichst vieler Fremd-

7 Interview mit Prof. Harald Lesch, Internet http://www.astronomie.de/bibliothek/interview/ lesch/lesch.htm. 
wörter, Kurven, Grafiken und Falschfarbenbilder, sondern unter anderem darin, wie jemand sein Wissen an einen Zuschauerkreis bringt - zum Beispiel eben mit einer genialen Sprache. Unter den vielen Wissenschaftsmoderatoren des heutigen Fernsehens ist Lesch sicher einer, bei dem es sich lohnt, mehr als nur eine Viertelstunde hinzuschauen und -zuhören.

\section{Faszination Natur - Streifzug mit Jakob Forster durch Wälder, Wiesen und Weiher. CD-ROM, Fr. 48.-, erhältlich in allen Buchhandlungen und im Lehrmittelverlag des Kantons Zürich (www.lehrmittelverlag.com)}

Vierzig Jahre lang hat der heute pensionierte Winterthurer Biologielehrer Jakob Forster Amphibien und Insekten beobachtet und sie ständig mit Fotoapparat und Filmkamera verfolgt. Dabei hatte er ein wichtiges Ziel: Den jüngeren Generationen die Vielfalt in Fauna und Flora, die es nach wie vor in unseren Lebensräumen gibt, näherzubringen und ihnen - falls sie dazu auch bereit sind - die Augen zu öffnen.

Für mich wurde die Sammlung gleichsam zum Gradmesser über den Zustand der Natur. Deshalb habe ich mich auch entschlossen, diese Beobachtungen an die nächste Generation weiterzugeben. Der heutigen Jugend, die Naturvorgänge oftmals nur vom Fernsehen kennt, möchte ich mit dieser DVD (CD-ROM. Red.) zeigen, wie schön, vielfältig und spannend unsere Natur ist. ${ }^{8}$

«Augen auf - das Schöne lebt so nah» könnte man manche seiner Schnappschüsse auch nennen.

Abb.3.Pechlibelle. @ Jakob Forster.

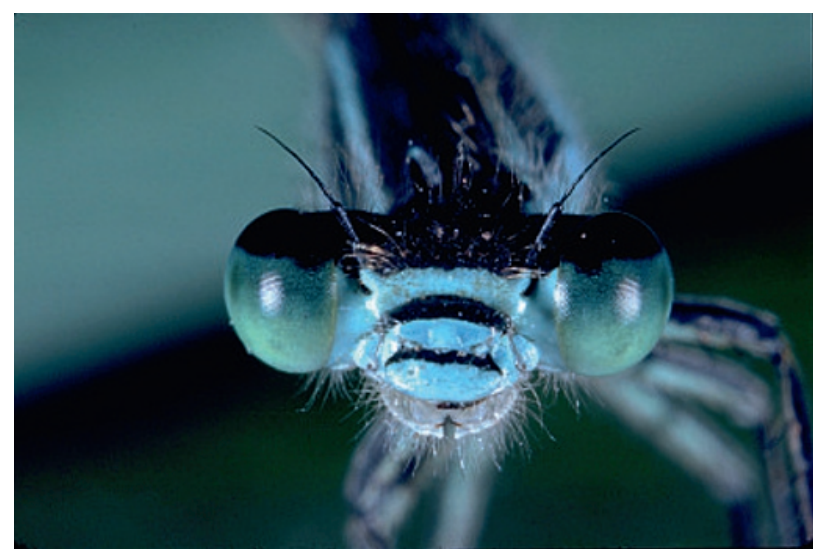

Was Forster um Winterthur herum, aber auch im Pfynwald im Kanton Wallis und in ausgewählten südlichen Ländern und Inseln wie Costa Rica, Rhodos oder Mallorca gefunden hat, ist eine Fundgrube punkto einheimi-

8 Aus Einleitung «Faszination Natur - Streifzüge mit Jakob Forster durch Wälder, Wiesen und Weiher», ISBN 978-3-033-00612-6. 
scher Fauna und Flora. So gelang es ihm, im Wallis Holzwespen zu filmen, die mit ihrem langen Sägestachel ein Loch in einen Baumstamm für die Eiablage bohrten, oder er sah Schornstein-Lehm- und Töpferwespen beim Bau ihrer arttypischen Behausungen zu, stunden-, wenn nicht gar tagelang. Forster scheint immer zum richtigen Zeitpunkt die Filmkamera oder den Fotoapparat angesetzt zu haben ...

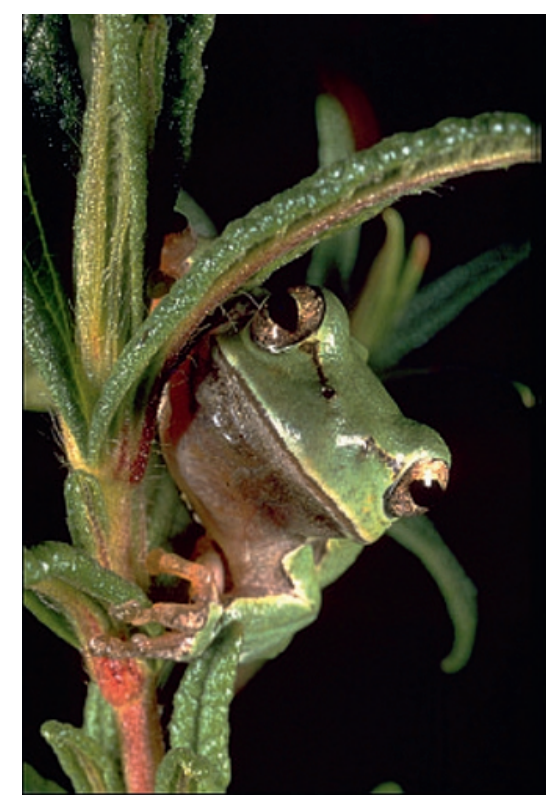

Abb.4.Laubfrosch. @ Jakob Forster.

Seine Sujets sind vielfältig: Sich häutende Libellen und sich verwandelnde Schmetterlingsraupen; Hummeln, die sich um eine Ragwurzblüte balgen, weil sie in ihr vermeintlich eines ihrer Weibchen sehen; Blattschneiderameisen, die unablässig der Pheromonspur folgend von Bäumen abgeschnittene Blätter unter die Erdoberfläche in ihre Bauten transportieren.

Forsters Geduld macht sich bezahlt. Der Betrachter, der sich, von einer einfachen Navigation angeleitet, durch die CD-ROM klickt, begegnet einer Fülle von hervorragenden Aufnahmen. Gerade für Entomologen ist «Faszination Natur» eine wichtige Sammlung. Im Wallis konnte Forster beispielsweise den Heuschreckenjäger, die Bläulingsraupen und die Wächterameisen filmen, sowie die Kreisel- und Sandwespen verfolgen. An all diesen unscheinbaren Tierchen geht der Spaziergänger achtlos vorbei, sofern er eben nicht die Augen öffnet.

Forsters CD-ROM beinhaltet eine Vielfalt der noch existierenden Flora um Winterthur herum. So wachsen in den wenigen noch vorhandenen Magerwiesen unter vielen anderen auch der Türkenbund, das Scharbockskraut sowie Manns- und Fuchs-Knabenkräuter. Forster hat typische Magerwiesenblumen wie die Wiesen-Flockenblume, die Rapunzel, den Wundklee, aber 
auch die gemeine Kreuzblume, die grossblütige Brunelle oder den zottigen Klappertopf fotografiert. Zusammen mit vielen ökologisch fundierten Erklärungen gibt er diesen prächtigen Blumen einen wohlverdienten Platz auf seiner CD-ROM.

Abb. 5. Knabenkraut. (C) Jakob Forster.

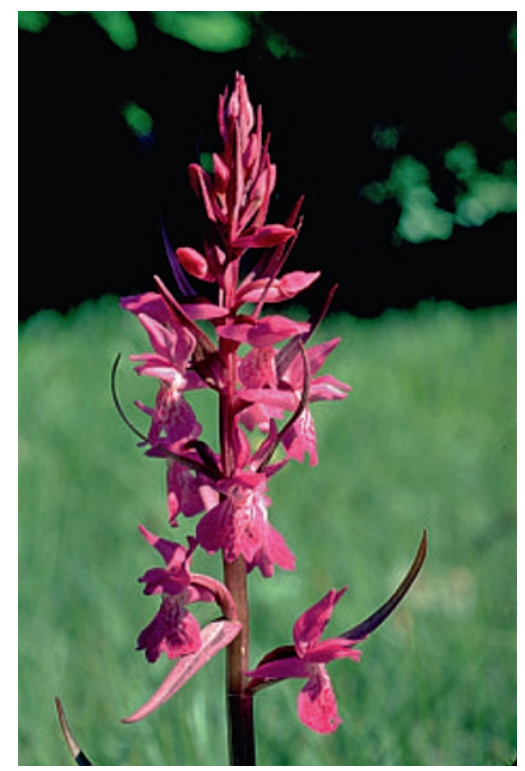

Unsichtbares sichtbar machen. Auch hier gelingt es einem begeisterten Naturliebhaber dem mit der Natur weniger vertrauten, aber trotzdem offenen Stadtbewohner Schönheiten zu zeigen, die plötzlich eines Tages - wenn wir nicht Sorge dazu tragen - nicht mehr da sein könnten.

\section{Eine Frage der Perspektive}

Die drei Beispiele haben eines fast exemplarisch gezeigt: Natur in den Medien - und mögen diese noch so vielfältig sein - ist nicht einfach, wie sie erscheint, sondern sie erscheint so, wie sie inszeniert worden ist. Erst durch die Wahl der Perspektive wird die Natur zu dem, was wir «Natur» nennen so die These in diesem Bericht. An und für sich könnte Natur ja auch langweilig dargestellt werden, brutal, zerstörerisch, liebevoll, logisch oder gar unverständlich. Alle drei Exponenten greifen hier auch zu unterschiedlichen Modellen, um «ihre Natur» zu präsentieren. Der mit dem REM arbeitende Biologe produziert eine fremd-schaurige Ästhetisierung mit Elektronenbildern. Er trocknet seine «Natur» ein, bedampft sie, beschiesst sie mit Elektronen und koloriert anschliessend die Bilder neu - und zwar so, wie er sich die Natur vorstellt. Im anderen Fall ist es das Schulzimmer, das zur Naturinszenierung herangezogen wird; die Welt soll mit Worten, Formeln und einer 
Kreidetafel gebändigt werden, damit sie so für den Zuschauer «logisch»erscheint. Und im dritten Fall ist es die klassische Herangehensweise des «Sammlers», der die Natur entdeckt, das Ganze aber unter dem modern ökologischen Modell der «Vielfalt» präsentiert. Alle drei Autoren arbeiten mit dem Modell, dass Natur etwas Faszinierendes ist. Und so spielen hier die Medien (ob elektronisch, technisch oder Print) nicht nur ihre grosse Rolle als Multiplikatoren aus, sondern beeinflussen mit dieser Rolle die Wissenschaft nachhaltig. Letztlich sind es Einschaltquoten und Leserzahlen, die auch in der Wissenschaft zum kaum mehr wegzudenkenden Element werden. Wissenschaft erhält mit solchen Inszenierungen in der Öffentlichkeit das zurück, was ihr gebührt: Faszination. 